\title{
VARIACIÓN MORFOLÓGICA ASOCIADA A LOS PATRONES DE COLOR DE LA GARGANTA, EN LAGARTIJAS SCELOPORUS HORRIDUS HORRIDUS (SAURIA: PHRYNOSOMATIDAE)
}

\author{
María Guadalupe BUSTOS ZAGAL, ${ }^{1}$ Rubén CASTRO-FRANCO, ${ }^{1 *}$ JaVIER \\ MANJARREZ $S^{2} \&$ VÍCTOR FAJARDO GUADARRAMA ${ }^{3}$ \\ ${ }^{1}$ Laboratorio de Herpetología, Departamento de Biología Animal, Centro de Investigaciones \\ Biológicas, Universidad Autónoma del Estado de Morelos, Av. Universidad 1001, Chamilpa 62209, \\ Cuernavaca, Morelos, México. (MGBZ): < bustosgu@uaem.mx>, (RCF): <castro@uaem.mx> \\ ${ }^{2}$ Centro de Investigación en Recursos Bióticos, Universidad Autónoma del Estado de México, \\ Toluca Estado de México, CP 50000, México. \\ ${ }^{3}$ Laboratorio de Ecología de la Conducta, Facultad de Ciencias, Universidad Autónoma del Estado de \\ México, Toluca Estado de México, CP 50000, México \\ *Envío de correspondencia.
}

Bustos Zagal, M. G., Castro-Franco, R., Manjarrez S., J. \& Fajardo Guadarrama, V. 2014. Variación morfológica asociada a los patrones de color de la garganta, en lagartijas Sceloporus horridus horridus (Sauria: Phrynosomatidae). Acta Zoológica Mexicana (n. s.), 30(2): 357-368.

RESUMEN. El dimorfismo sexual, es un fenómeno común en lagartijas del género Sceloporus, lo que permite identificar diferencias en el tamaño de cuerpo, y diseño morfológico, entre machos y hembras. Lo más común es que ocurran variaciones en el color disociadas de la morfología. Utilizando ANDEVA de una vía y análisis discriminante, obtuvimos diferencias significativas en seis caracteres morfológicos en correlación con color de la garganta; lo que permite reconocer tres diferentes fenotipos (amarillo, azul y naranja). Sin embargo, no observamos diferencias en el uso de microhábitats. Los machos con garganta de color azul (44.35\%) fueron los más grandes y robustos, y tienen similar abundancia que los machos con garganta de color amarillo (48.5\%). Los machos con garganta naranja solo representaron el $8 \%$ del total. Sugerimos que los mecanismos de selección de machos por parte de las hembras y las oportunidades de realizar cópula, por parte de los tres morfos, pueden inducir variaciones en la inversión reproductora entre localidades. Sugerimos que las características de cada morfo, son un factor importante a considerar en estudios sobre inversión reproductoras de las especies.

Palabras clave: Coloración, morfología, machos, Sceloporus horridus.

Bustos Zagal, M. G., Castro-Franco, R., Manjarrez S., J. \& Fajardo Guadarrama, V. 2014. Morphological variation associated to color patterns of the throat in lizards Sceloporus horridus horridus (Sauria: Phrynosomatidae). Acta Zoológica Mexicana (n. s.), 30(2): 357-368.

Recibido: 10/07/2013; aceptado: 19/02/2014. 
ABSTRACT. Sexual dimorphism is a common phenomenon in lizards of the genus Sceloporus, this allows to identify differences in body size, and morphological design between males and females. Usually a variation occurs in color dissociated from the morphology. Using one-way ANOVA and discriminant analysis, we obtained significant differences in six morphological traits correlated with throat color, allowing the recognition of three different phenotypes (yellow, blue and orange). However, we did not observe differences in microhabitat use. Males with blue throat $(44.35 \%)$ were the largest and the most robust, and have a similar abundance as males with yellow throat (48.5\%). Males with orange throats only represented $8 \%$ of the total. We suggest that males' selection mechanisms by females and the opportunities for copulation, by the three morphs, can induce changes in reproductive investment between locations. We suggest that morphological characteristics are an important factor to consider in studies on reproductive investment of species.

Key words: Coloration, morphology, males, Sceloporus horridus.

\section{INTRODUCCIÓN}

El dimorfismo sexual es un fenómeno común en el reino animal que permite identificar diferencias en el tamaño de cuerpo y diseño de estructuras entre machos y hembras (Anderson 1994). Usualmente se manifiesta como cambios de color en varias regiones del cuerpo, por el efecto de hormonas que circulan en el torrente sanguíneo (Cox et al. 2005).

Las lagartijas del genero Sceloporus son notables por su acentuado dimorfismo sexual; los machos generalmente tienden a ser más grandes que las hembras (Fitch 1978), y en la época de reproducción desarrollan diversos patrones de color en la región de la garganta, vientre y dorso, que regularmente se asocian con la madurez sexual y el estatus social (Carpenter 1995, Lemos-Espinal et al. 1996).

El color también funciona como un signo confiable de la habilidad para obtener alimentos de buena calidad y componentes específicos para desarrollar el color como carotenoides (Cooper \& Greenberg 1992). Esta característica se utiliza para enviar señales hacia machos rivales, desafiar a depredadores potenciales y atraer a las hembras (Whiting et al. 2003).

En estudios previos se ha observado que las hembras de Sceloporus undulatus, Urosaurus ornatus y Liolaemus tenuis, seleccionan a los machos en función de los despliegues agonísticos, los patrones de color y el efecto combinado de características fenotípicas como la masa del cuerpo, tamaño de la cabeza y color de la cola (Cooper Jr. \& Burns 1987, Hamilton \& Sullivan 2005, Vidal et al. 2007). Asimismo, se ha observado que los machos con un sistema elaborado de señales de color, tienen acceso a territorios de mejor calidad y pueden aparearse más fácilmente (Anderson 1994).

Los machos de lagartijas endémicas de México Sceloporus $h$. horridus, con amplia distribución en la región centro sur de Morelos y cuenca del Río Balsas (CastroFranco \& Bustos 2003), se diferencian de las hembras por una serie de características sexuales como un sistema variable de colores en la región de la garganta y un tamaño 
de cuerpo variable, lo que hace posible reconocer machos con diversos patrones de color y tamaño dentro de una misma población. Este polimorfismo ha sido observado en varias lagartijas phrynosomátidas (Sinervo et al. 2000, Hamilton \& Sullivan 2005) y tiene implicaciones en los proceso de especiación mórfica (Smith 1962, Gray \& McKinnon 2007, Corl et al. 2010) por la pérdida selectiva de morfos.

En este trabajo presentamos evidencias sobre la variación en los patrones de color de la garganta de machos adultos de $S$. horridus horridus y como estos patrones de color están asociados a variaciones en la morfología; asimismo, presentamos información sobre el uso de diferentes microhábitats entre los morfos.

\section{MATERIAL Y MÉTODOS}

Utilizamos una base de datos de 363 machos adultos de la colección de anfibios y reptiles de la Universidad Autónoma del Estado de Morelos (CARUM); los cuales provienen de 67 localidades de la región centro sur de Morelos, en áreas de selva baja subperennifolia espinosa, selva baja caducifolia, selva mediana subcaducifolia, bosque caducifolio espinoso de Prosopis y partes bajas de pino-encino (Castro-Franco \& Bustos 2003). De cada ejemplar en vivo, se obtuvieron registros del color de la garganta y de la longitud hocico-cloaca (LHC), largo de la cabeza (LC, distancia lineal entre el borde del hocico y el borde anterior del tímpano), alto de la cabeza (ALC, distancia lineal entre el borde lateral de la garganta y la región parietal), ancho de cabeza (ANC, distancia lineal entre los dos bordes de la cabeza a la altura de la región postocular), longitud de la extremidad anterior (LEA, distancia lineal que se obtiene al sumar la longitud del humero, longitud del radio, longitud del metacarpo y longitud de las falanges del dedo más largo) y longitud de la extremidad posterior (LEP, distancia lineal que se obtiene al sumar la longitud del fémur, longitud de la tibia, longitud del metatarso y longitud de las falanges del dedo más largo) (Hamilton \& Sullivan 2005, Herrel et al. 2007). Todas las medidas fueron registradas con un vernier digital $(0.01 \mathrm{~mm})$.

Las diferencias en los seis caracteres descritos entre los morfos (color de la garganta), fueron evaluadas con Análisis de Varianza (ANDEVA) de una vía y pruebas post hoc de Duncan. Adicionalmente, se utilizó un Análisis Discriminante para reducir la probabilidad de error al clasificar los morfos en función de sus características individuales. La función discriminante por ser una prueba multivariada, permite identificar el conjunto de variables que mejor caracterizan a los distintos morfos (Johnson 2000).

Las relaciones alométricas de los morfos fueron estimadas con análisis de regresión lineal múltiple, utilizando la longitud hocico-cloaca (LHC) como la variable independiente; y el tamaño de la cabeza (LC, ALC, ANC) y las extremidades (LEA, LEP) como las variables dependientes. 
El uso diferente de los distintos microhábitats entre los morfos, fue estimado con una prueba de Chi-cuadrada $\left(\chi^{2}\right)$ utilizando los datos de frecuencia de avistamiento de cada morfo sobre sitios específicos como suelo, rocas y árboles.

\section{RESULTADOS}

Descripción de los Morfos. El análisis de las características fenotípicas de las lagartijas examinadas mostró que existen tres diferentes morfos. Los machos de Sceloporus $h$. horridus tienen gargantas de color amarillo, azul y naranja (Figs. 1 y 2). Los machos con garganta de color azul, fueron los más grandes y robustos, y tuvieron similar abundancia (44.35\%) que los machos con garganta de color amarillo (48.5\%). Los machos con garganta de color naranja fueron relativamente escasos y solo representaron el $8 \%$ del total.

También existen parches de color en las escamas de la región ventral, la región dorso lateral del cuerpo y en el dorso de las extremidades anteriores y posteriores. De acuerdo con el ANDEVA y la prueba de Duncan, los morfos son reconocibles entre sí por variaciones significativas en los siguientes caracteres: longitud hocico-cloaca; largo, ancho y alto de la cabeza; y longitud de las extremidades anteriores y posteriores (Cuadro 1). Los machos del morfo azul son los más grandes de los tres, por tener la mayor longitud hocico-cloaca (LHC $91.97 \mathrm{~mm} \pm 10.18, n=161$ ), lo mismo que la longitud de la cabeza (LC $24.30 \mathrm{~mm} \pm 2.71, n=161$ ), la altura de la cabeza (ALC $14.96 \pm 2.48, n=161$ ) y las extremidades anteriores (LEA $34.57 \mathrm{~mm} \pm 4.40, n=161$ ) y posteriores (LEP $50.79 \mathrm{~mm} \pm 6.30, n=161$ ). Después se encuentran los machos del morfo naranja (LHC $83.54 \pm 7.82, n=26$ ); y los del morfo amarillo (LHC $81.93 \pm$ $7.45, n=176$ ), que tuvieron las medidas del cuerpo más pequeñas (Cuadro 1).

El análisis discriminante mostró que los caracteres largo $(\lambda=0.6186, P=0.0065)$ $\mathrm{y}$ alto $(\lambda=0.6407, P=0.0000)$ de la cabeza, y longitud de las extremidades anteriores $(\lambda=0.6126, P=0.0374)$ y posteriores $(\lambda=0.6193, P=0.0052)$ son los caracteres que mejor describen las diferencias entre los morfos identificados. Los valores Lambda de Wilks $(\lambda)$ fueron significativos para estos caracteres (Cuadro 2, Fig. 3).

Relaciones alométricas. Existen relaciones significativas entre la longitud hocicocloaca (LHC) de cada morfo y el tamaño de la cabeza (LC, ALC, ANC) y las extremidades (LEA, LEP) (Cuadro 3). La relación LHC/LC del morfo naranja, fue el único valor significativo $\left(\beta_{1}=0.442, t=0.228, P=0.032\right)$.

Uso de hábitat. Por el número de datos disponibles, no fue posible analizar el uso de micro hábitats del morfo naranja. Sin embargo, el análisis de los datos de los morfos de garganta amarilla y garganta azul reveló que usan tres distintos microhábitats. El $60 \%$ de los ejemplares de garganta amarilla fueron observados en árboles, el 28.88 $\%$ en rocas y el $11.11 \%$ en suelo. De manera similar, el $51.16 \%$ de los ejemplares de garganta azul fueron observados en árboles, el $39.53 \%$ en rocas, y el $9.30 \%$ en suelo. 

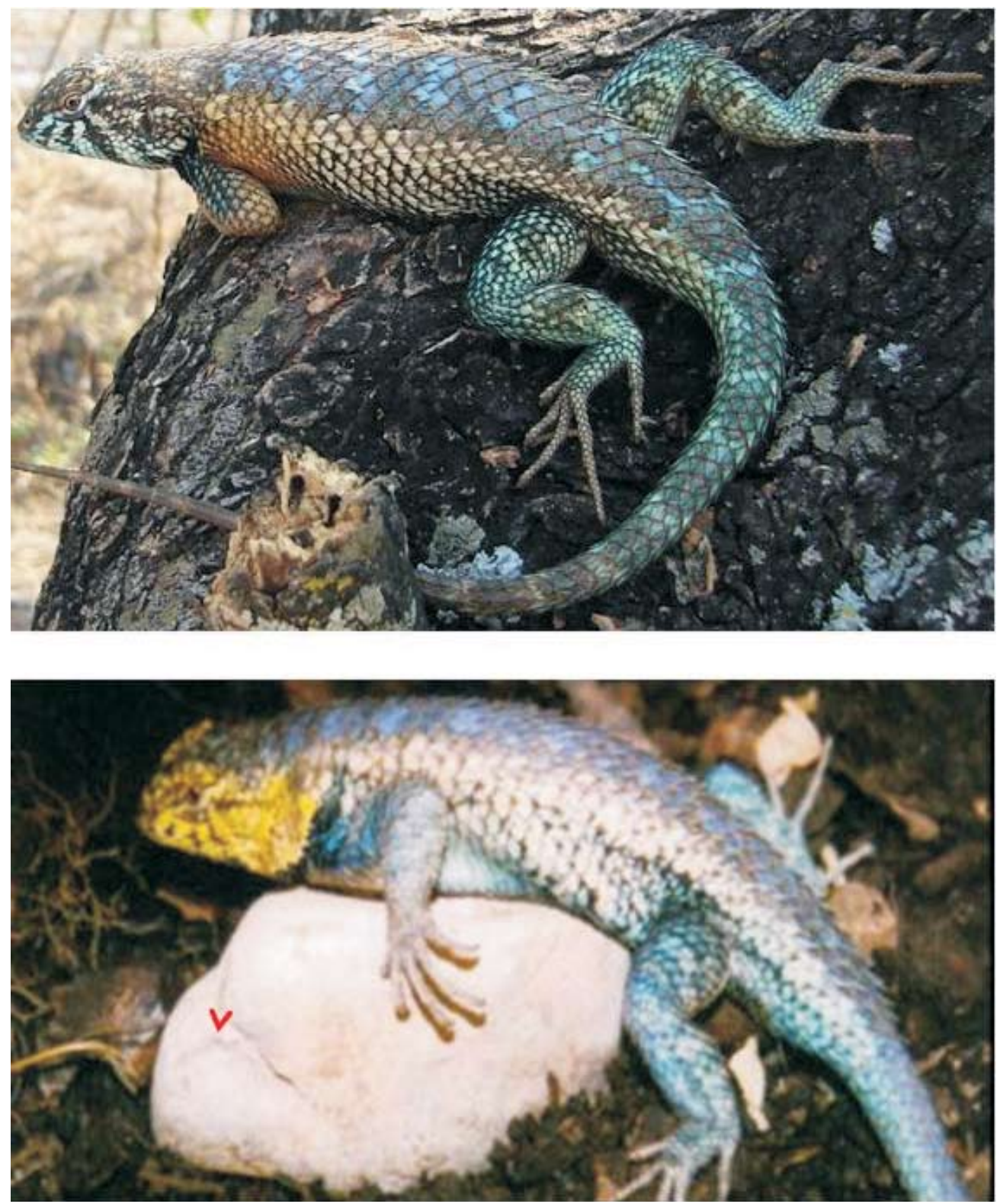

Figura 1. Coloración dorsal de machos de Sceloporus h. horridus. Arriba el morfo de color azul y abajo el morfo de color amarillo. 
Bustos et al.: Variación morfológica, patrones de color, Sceloporus h. horridus
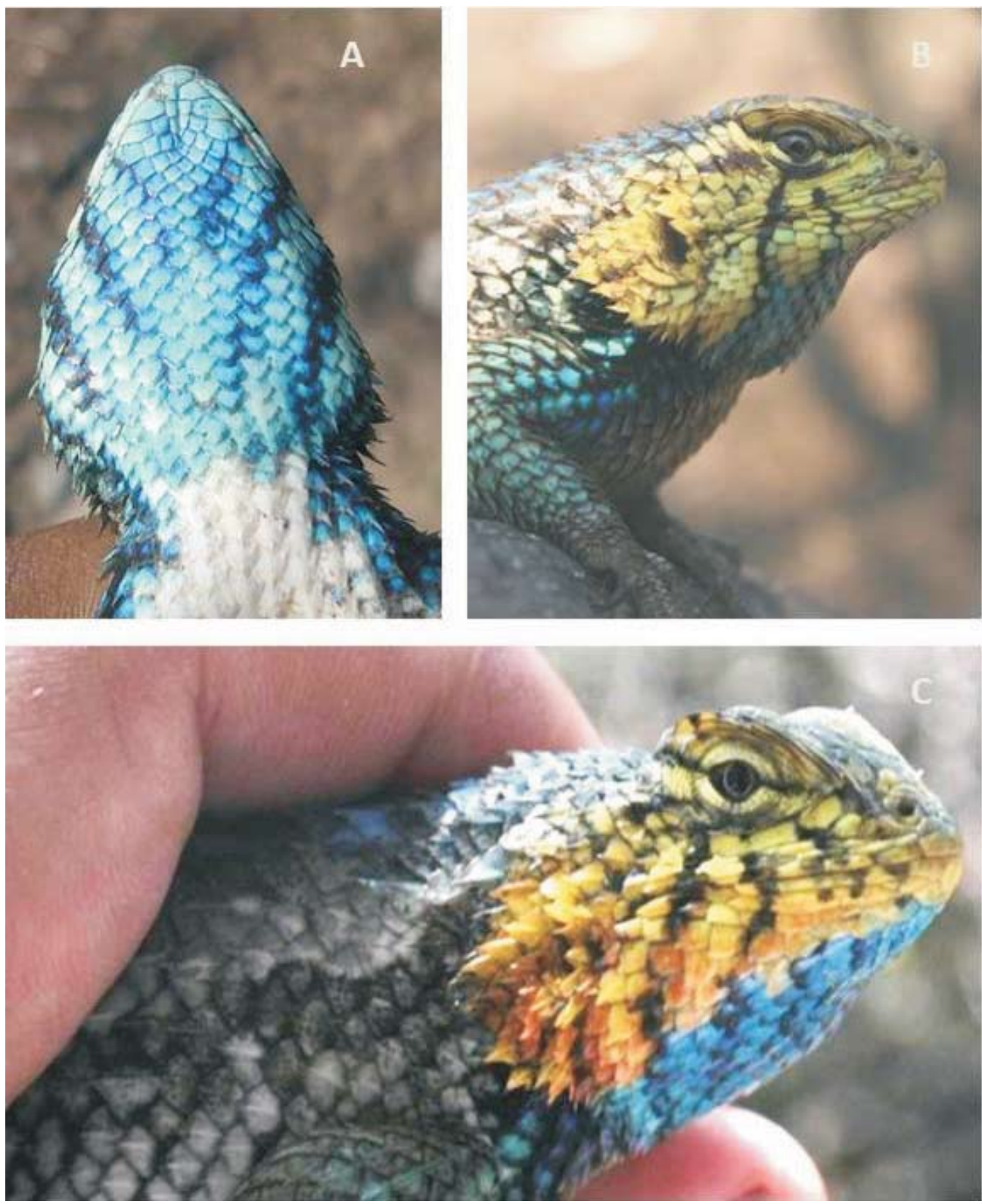

Figura 2. Variaciones en el patrón de color de la garganta. A, macho con garganta de coloración azul. $\mathrm{B}$, macho con garganta de color amarillo. $\mathrm{C}$, machos con garganta de color rojo. 
Cuadro 1. Variación morfológica entre los tres patrones de color de la garganta de lagartijas Sceloporus $h$. horridus del Estado de Morelos. Medidas en mm, promedio \pm desviación estándar, entre paréntesis valor mínimo y máximo, el asterisco indica diferencias significativas.

\begin{tabular}{|c|c|c|c|c|}
\hline Caracteres & $\begin{array}{c}\text { Morfo Amarillo } \\
\text { (Mam) } \\
n=176\end{array}$ & $\begin{array}{c}\text { Morfo Azul } \\
\text { (Maz) } \\
n=161\end{array}$ & $\begin{array}{c}\text { Morfo Naranja } \\
\quad(\mathrm{Mn}) \\
n=26\end{array}$ & $\begin{array}{c}\text { Prueba de Duncan } \\
\text { entre los morfos }\end{array}$ \\
\hline $\begin{array}{c}\text { Longitud } \\
\text { Hocico-Cloaca } \\
\text { (LHC) }\end{array}$ & $\begin{array}{c}81.93 * \pm 7.45 \\
(71.0-100.8)\end{array}$ & $\begin{array}{c}91.97 * \pm 10.18 \\
(71.5-115.9)\end{array}$ & $\begin{array}{c}83.54 * \pm 7.82 \\
(71.33-98.3)\end{array}$ & $\begin{array}{c}\text { Mam vs. Maz } \\
D=0.0000 \\
\text { Maz vs. Mn } \\
D=0.0000\end{array}$ \\
\hline $\begin{array}{l}\text { Largo de la } \\
\text { cabeza } \\
\text { (LC) }\end{array}$ & $\begin{array}{c}21.33 * \pm 2.20 \\
(16.7-28.3)\end{array}$ & $\begin{array}{c}24.30 * \pm 2.71 \\
(16.6-30.7)\end{array}$ & $\begin{array}{c}21.92 * \pm 1.84 \\
(18.8-25.5)\end{array}$ & $\begin{array}{c}\text { Mam vs. Maz } \\
D=0.0000 \\
\text { Maz vs. Mn } \\
D=0.0000\end{array}$ \\
\hline $\begin{array}{l}\text { Alto de la cabeza } \\
\text { (ALC) }\end{array}$ & $\begin{array}{l}11.91 * \pm 1.90 \\
(8.1-17.1)\end{array}$ & $\begin{array}{l}14.96^{*} \pm 2.48 \\
(9.8-23.7)\end{array}$ & $\begin{array}{c}13.07 * \pm 2.00 \\
(10.5-20.1)\end{array}$ & $\begin{array}{c}\text { Mam vs. Maz } \\
D=0.0000 \\
\text { Mam vs. Mn } \\
D=0.0033 \\
\text { Maz vs. Mn } \\
D=0.0000\end{array}$ \\
\hline $\begin{array}{c}\text { Ancho de la } \\
\text { cabeza } \\
\text { (ANC) }\end{array}$ & $\begin{array}{c}17.74 * \pm 4.70 \\
(13.6-74.4)\end{array}$ & $\begin{array}{c}20.83 * \pm 2.91 \\
(12.8-27.2)\end{array}$ & $\begin{array}{c}18.57 * \pm 1.96 \\
(15.3-22.5)\end{array}$ & $\begin{array}{c}\text { Mam vs. Maz } \\
D=0.0000 \\
\text { Azul vs. Mn } \\
D=0.0011\end{array}$ \\
\hline $\begin{array}{l}\text { Longitud de } \\
\text { la extremidad } \\
\text { anterior } \\
\text { (LEA) }\end{array}$ & $\begin{array}{c}30.40 * \pm 3.51 \\
(20.0-39.2)\end{array}$ & $\begin{array}{c}34.57 * \pm 4.40 \\
(24.4-45.3)\end{array}$ & $\begin{array}{c}29.88^{*} \pm 3.47 \\
(24.7-41.0)\end{array}$ & $\begin{array}{c}\text { Mam vs. Maz } \\
D=0.0000 \\
\text { Maz vs. Mn } \\
D=0.0000\end{array}$ \\
\hline $\begin{array}{l}\text { Longitud de } \\
\text { la extremidad } \\
\text { posterior } \\
\text { (LEP) }\end{array}$ & $\begin{array}{c}43.38 * \pm 5.15 \\
(29.2-56.0)\end{array}$ & $\begin{array}{c}50.79 * \pm 6.30 \\
(34.2-68.3)\end{array}$ & $\begin{array}{c}29.88^{*} \pm 0.47 \\
(24.7-41.0)\end{array}$ & $\begin{array}{c}\text { Mam vs. Maz } \\
D=0.0000 \\
\text { Maz vs. Mn } \\
D=0.0000\end{array}$ \\
\hline
\end{tabular}

Sin embargo, no existen diferencias significativas en el uso de microhábitats entre los dos morfos $\left(\chi^{2}=1.11, P=0.05, g l=1\right)$.

\section{DISCUSIÓN}

La variación en los patrones de color de la garganta (amarillo, azul, naranja) que se ha observado en los machos de Sceloporus h. horridus, y su relación con la morfología del cuerpo, revelan un acentuado dimorfismo sexual en relación con las hembras. Esto contrasta con lo registrado previamente por Valdéz-González \& Ramírez-Bautista (2002), quienes no encontraron dimorfismo en esta especie. Una posible explicación 
Bustos et al.: Variación morfológica, patrones de color, Sceloporus h. horridus

Cuadro 2. Valores de lambda producto del análisis discriminante de seis caracteres de Sceloporus h. horridus. Las variables que mejor separan a los tres morfos (amarillo, azul, naranja) son Largo de la cabeza (LC), Alto de la cabeza (ALC), Longitud de la extremidad anterior (LEA), Longitud de la extremidad posterior (LEP). El asterisco indica diferencias significativas.

\begin{tabular}{|c|c|c|c|c|}
\hline Caracteres & $\begin{array}{c}\text { Wilks' } \\
\text { Lambda } \\
(\lambda)\end{array}$ & $\begin{array}{c}\text { Lambda } \\
\text { Parcial } \\
(\lambda)\end{array}$ & $\begin{array}{l}F \text {-remoción } \\
\quad(2,351)\end{array}$ & $\begin{array}{l}\text { Nivel de } \\
\text { la } P\end{array}$ \\
\hline $\begin{array}{l}\text { Longitud Hocico-Cloaca } \\
\text { (LHC) }\end{array}$ & 0.6027 & 0.9977 & 0.3954 & 0.6736 \\
\hline Largo de la cabeza (LC) & 0.6186 & 0.9722 & 5.0945 & $0.0065^{*}$ \\
\hline Alto de la cabeza (ALC) & 0.6407 & 0.9386 & 11.6592 & $0.0000 *$ \\
\hline Ancho de la cabeza (ANC) & 0.6033 & 0.9968 & 0.5724 & 0.5646 \\
\hline $\begin{array}{l}\text { Longitud de la extremidad } \\
\text { anterior (LEA) }\end{array}$ & 0.6126 & 0.9817 & 3.3144 & $0.0374 *$ \\
\hline $\begin{array}{l}\text { Longitud de la extremidad } \\
\text { posterior (LEP) }\end{array}$ & 0.6193 & 0.9710 & 5.3215 & $0.0052 *$ \\
\hline
\end{tabular}

para esta diferencia es que los autores referidos pudieron haber medido machos del morfo con garganta amarilla, cuyo tamaño de cuerpo registrado en este trabajo (81.93 $\mathrm{mm} \pm$ D. E. 7.45, $n=176,71.0-100.8 \mathrm{~mm}$ ) fue similar al observado en hembras (LHC $=81.8 \mathrm{~mm} \pm$ D. E. $1.8, n=34,60.0-100.0 \mathrm{~mm}$, Valdéz-González \& Ramírez-Bautista 2002). Esto sugiere que la morfología de los machos con garganta amarilla, es similar a las hembras como ha sido observado previamente en machos de Uta stansburiana (Sinervo \& Lively 1996). El elevado porcentaje de machos del morfo amarillo en la población de Morelos (48.5\%) dan soporte a esta posible explicación.

A diferencia de lo esperado, los machos con garganta de color azul fueron los más grandes, en comparación con los machos de gargantas color amarillo o naranja, lo cual contrasta con lo observado previamente en otras especies. En Urosaurus ornatus y Uta stansburiana (Hover 1985, Sinervo \& Lively 1996) los machos de color naranja suelen ser los más grandes y agresivos, y asimismo defienden territorios grandes. Sin embargo, de acuerdo con Wiens (2000), en phrynosomátidos no hay una asociación consistente entre la conducta y la morfología. Por ahora, no se dispone de información sobre la conducta y defensa del territorio asociada a los morfos que aquí se describen; no obstante, un estudio sobre este tema está en progreso.

Sugerimos que debido a la variación observada en este trabajo, $S$. horridus horri$d u s$ es un buen modelo de estudio que permitiría probar varias hipótesis sobre patrones de color y aptitudes morfológicas relevantes (fitness) (Langkilde \& Boronow 2010).

Las variaciones en el tamaño de la cabeza (LC, ANC, ALC) son comunes en los machos de varias especies de saurios (Polychrotidae: Anolis carolinensis-Herrel et al.2007, Lacertidae: Podarcis carbonelli- Kaliontzopoulou et al. 2008, Sphenodonti- 


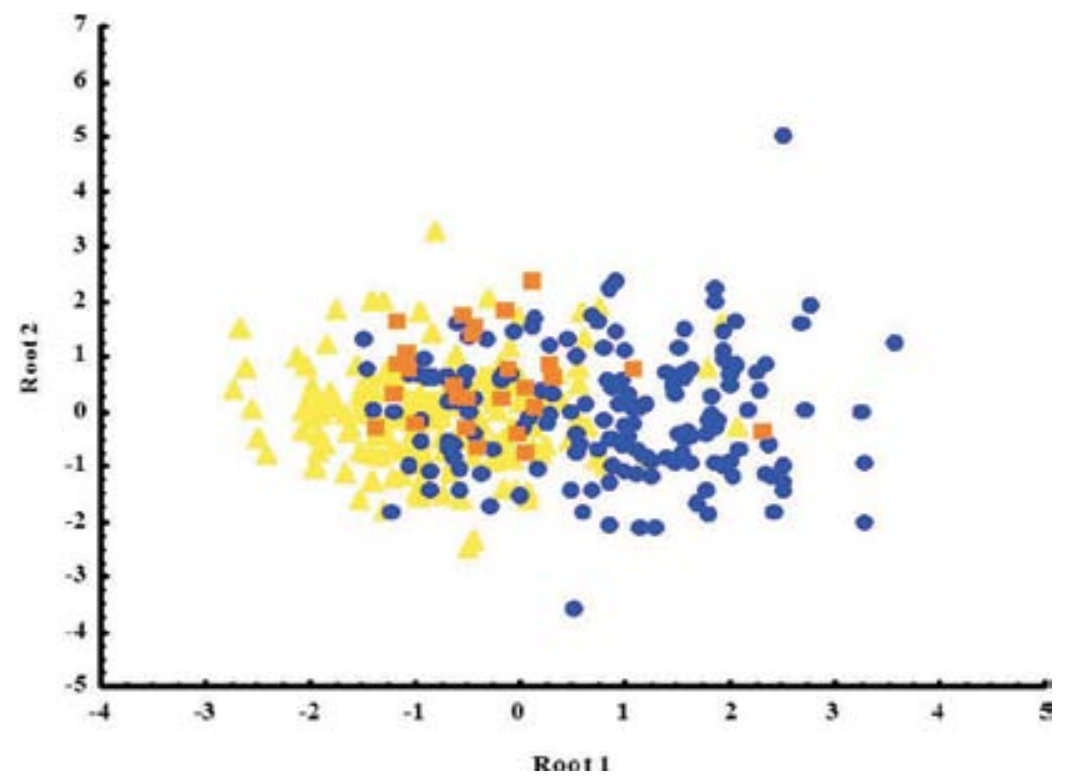

Figura 3. Gráfico del análisis discriminante entre machos de Sceloporus h. horridus donde se separan los tres morfos amarillo, azul y naranja. Los valores de lambda se muestran en el cuadro 2.

dae: Sphenodon punctatus- Herrel et al.2009, Agamidae: Japalura swinhonis- Kuo et al.2009, Iguanidae: Sauromalus ater- Lappin et al. 2006) y generalmente están asociadas con la fuerza de la mordedura y la capacidad de defensa del territorio, lo cual es de utilidad en los procesos de competencia con otros machos. Herrel et al. (2002) se ha referido a una relación poco consistente entre el diseño del cuerpo y el uso de hábitat en phrynosomatidos.

Los machos del morfo azul tuvieron las cabezas más grandes (más largas, más altas y más anchas) que los machos de los morfos amarillo y naranja, y lo mismo ocurrió con las extremidades anteriores y posteriores. Esto se explica porque esas variables se comportan como una función del tamaño del cuerpo (LHC). Lo que parece sugerir que los machos de color azul podrían tener una defensa más activa del territorio y en consecuencia mejores aptitudes-morfológicas relevantes (fitness) (Langkilde $\&$ Boronow 2010) que los morfos azul y naranja. En Sceloporus undulatus, el color de los machos se ha sugerido que funciona como una señal sexual (Langkilde \& Boronow 2010) porque es variable, es visible, está asociada con la dominancia (Smith \& John-Alder 1999), y por la habilidad de acceso a componentes precursores para el desarrollar color (Cooper \& Greenberg 1992).

Lo anterior revela que los patrones de color, están vinculados a varias característica biológicas de las especies, con efectos subsecuentes sobre la selección. La perma- 


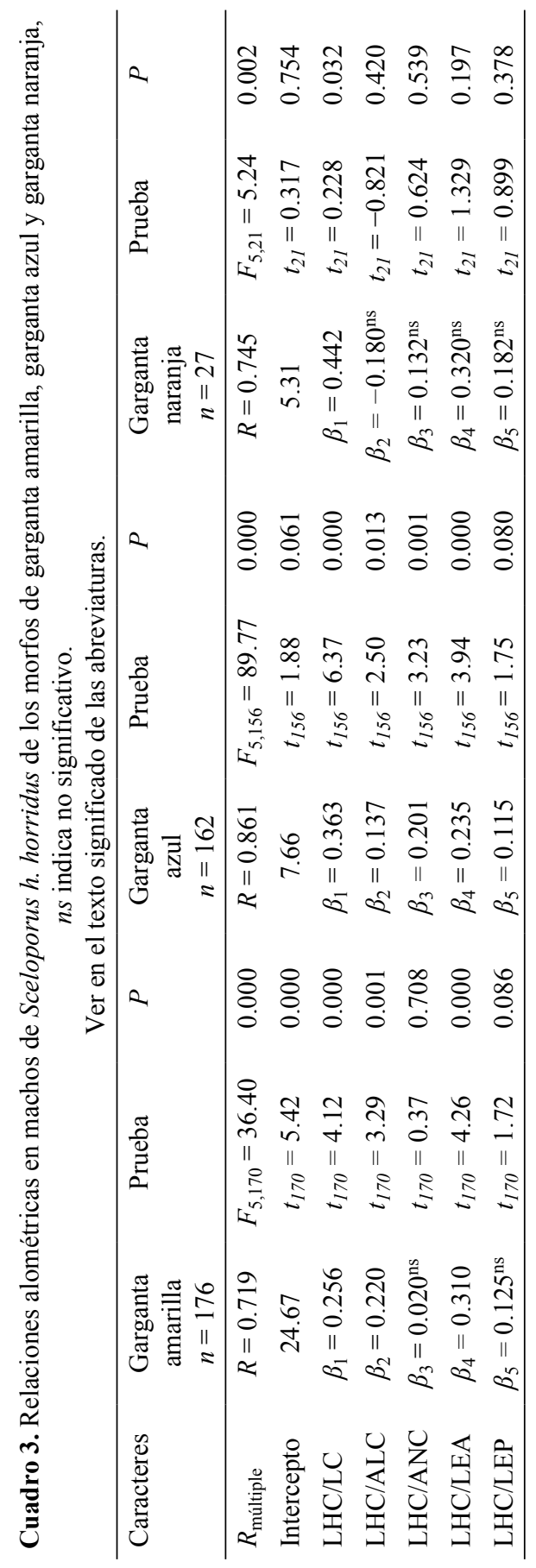


nencia de color asociada con la morfología constituye un caso típico de polimorfismo en machos, y contribuye a la discriminación sexual (Cooper \& Burns 1987) con implicaciones en la reproducción y los procesos de especiación. De acuerdo con Gross (1996) no todos los miembros de un sexo se comportan de la misma manera, los fenotipos dentro de los sexos tienen diferentes tácticas reproductoras, con diferentes comportamientos, color, tamaño y estrategias de defensa (Sinervo \& Lively 1996, Oliveira et al.2008). En consecuencia, la variación en el tamaño de la camada que ha sido observada en poblaciones de $S$. horridus horridus (Fitch 1985, Valdéz-González \& Ramírez-Bautista 2002) podría ser explicada en términos de una inversión reproductora diferente entre los tres morfos. Este tipo de variaciones han sido registradas previamente en varias especies de lagartijas (Sinervo \& Lively 1996, Oliveira et al. 2008).

\section{LITERATURA CITADA}

Anderson, M. 1994. Sexual Selection. Princeton University, Press, Princeton, NJ.

Carretero, M. A. 2002. Sources of colour pattern variation in Mediterranean Psammodromus algirus. Netherlands Journal of Zoology, 52: 43-60.

Carpenter, G. C. 1995. The ontogeny of a variable social badge: throat color development in tree lizards (Urosaurus ornatus). Journal of Herpetology, 29:7-13.

Castro-Franco, R. \& Bustos Z., M. G. 2003. Lagartijas de Morelos, México: distribución, hábitat y conservación. Acta Zoológica Mexicana (n.s.), 88: 123-142.

Cooper, W.E. \& Greenberg, N. 1992. Reptilian coloration and behavior. Pp. 298-422. In: B. C. Gans \& D. D. Crews (Eds). Biology of the Reptilia. Vol. 18. Physiology University of Chicago Press, Chicago.

Corl, A., Davis, A. R, Kuchta, S. R. Comendant, T. \& Sinervo, B. 2010. Alternative mating strategies and the evolution of sexual size dimorphism in the side-blotched lizard, Uta stansburiana: A population-level comparative analysis. Evolution, 64:79-96.

Cox, R. M., Skelly, S. L., Leo, A. \& John-Alder, H. B. 2005. Testosterone regulates sexually dimorphic coloration in the eastern fence lizard, Sceloporus undulates. Copeia, 3: 597-608.

Cooper Jr., W. E. \& Burns, N. 1987. Social significance of ventro lateral coloration in the fence lizard, Sceloporus undulatus. Animal Behaviour, 35: 526-532.

Fitch, H. S. 1978. Sexual size differences in the genus Sceloporus. University of Kansas Science Bulletin, 51: 441-461.

Fitch, H. S. 1985. Variation in clutch and litter size in new world reptiles. Miscellaneous Publication University of Kansas Museum of Natural History, 76: 1-75.

Gray, S. M. \& McKinnon, J. S. 2007. Linking color polymorphism maintenance and speciation. Trends in Ecology and Evolution, 22: 71-79.

Gross, M. R. 1996. Alternative reproductive strategies and tactics: Diversity within sexes. Trends in Ecology \& Evolution, 11: A92-A98.

Hamilton, P. S. \& Sullivan, B. K. 2005. Female mate attraction in ornate tree lizards, Urosaurus ornatus: a multivariate analysis. Animal Behaviour, 69: 219-224.

Herrel, A., Meyers, J. J. \& Vanhooydonck, B. 2002. Relations between microhabitat use and limb shape in Phrynosomatid lizards. Biological Journal of the Linnean Society, 77: 149-163

Herrel, A., Mcbrayer, L. D. \& Larson, P. M. 2007. Functional basis for sexual differences in bite force in the lizard Anolis carolinensis. Biological Journal of the Linnean Society, 91: 111-119. 
Bustos et al.: Variación morfológica, patrones de color, Sceloporus h. horridus

Herrel, A., Schaerlaeken, V., Moravec, J. \& Ross, C. F. 2009. Sexual shape dimorphism in Tuatara. Copeia, 4: 727-731.

Hover, E. L. 1985. Differences in aggressive behavior between two throat color morphs in a lizard, Urosaurus ornatus. Copeia, 4: 933-940.

Johnson, D. E. 2000. Métodos multivariados aplicados al análisis de datos. International Thomson Editores.

Kaliontzopoulou, A., Carretero, M. A. \& Llorente, G. A. 2008. Head shape allometry and proximate causes of head sexual dimorphism in Podarcis lizards: joining linear and geometric morphometrics. Biological Journal of the Linnean Society, 93: 111-124.

Kuo, C. Y., Lin, Y. T. \& Lin, Y. S. 2009. Sexual Size and Shape Dimorphism in an Agamid Lizard, Japalura swinhonis (Squamata: Lacertilia: Agamidae). Zoological Studies, 48: 351-361.

Langkilde, T. \& Boronow, K. E. 2010. Color as a signal: The relationship between coloration and morphology in male eastern fence lizards, Sceloporus undulatus. Journal of Herpetology, 44: 261-271.

Lappin A. K., Hamilton, P. S. \& Sullivan, B. 2006. Bite-force performance and head shape in a sexually dimorphic crevice-dwelling lizard, the common chuckwalla [Sauromalus ater (=obesus)]. Biological Journal of the Linnean Society, 88: 215-222.

Lemos-Espinal, J. A., Smith, G. H. \& Ballinger, R. E. 1996. Ventral blue coloration and sexual maturation in male Sceloporus gadoviae lizards. Journal of Herpetology, 30: 546-548.

Oliveira, R. F., Taborsky, M. \& Brockmann, H. J. (Eds.) 2008. Alternative Reproductive Tactics: An Integrative Approach. Cambridge University Press, New York.

Sinervo, B. \& Lively, C. M. 1996. The rock-paper-scissors game and the evolution of alternative male strategies. Nature, 380: 240-243.

Smith, J. M. 1962. Disruptive selection, polymorphism and sympatric speciation. Nature, 195: 60-62.

Smith, L. C. \& John-Alder, H. B. 1999. Seasonal specificity of hormonal, behavioral, and coloration responses to within- and between-sex encounters in male lizards (Sceloporus undulatus). Hormones and Behavior, 36: 39-52.

Valdéz-González, M. \& Ramírez-Bautista, A. 2002. Reproductive characteristics of the spiny lizards, Sceloporus horridus and Sceloporus spinosus (Squamata: Phrynosomatidae) form Mexico. Journal of Herpetology, 36: 36-43.

Vidal, M. A., Ortiz, J. C. \& Labra, A. 2007. Sexual and geographic variation of color patterns in Liolaemus tenuis (Squamata, Liolaeminae). Gayana, 71: 27-33.

Wiens, J. J. 2000. Decoupled evolution of display morphology and display behaviour in phrynosomatid lizards. Biological Journal of the Linnean Society, 70: 597-612.

Whiting, M. J., Nagy, K. A. \& Bateman, P. W. 2003. Evolution and maintenance of social status signaling badges: experimental manipulations in lizards, pp. 47-82. In: S. F. Fox, J.K. McCoy and T.A. Baird (Eds.). Lizard Social Behavior, Johns Hopkins University Press, Baltimore. 